\title{
Perspectiva dos enfermeiros sobre a assistência pré-natal no âmbito da Estratégia Saúde da Família
}

Nurses' perspective on prenatal care under the Family Health Strategy

Perspectiva de los enfermeros sobre la asistencia prenatal en el ámbito de la Estrategia Salud de la Familia

\section{Luana Carla dos Santos Nascimento ${ }^{1}$, Maria Rejane Ferreira da Silva ${ }^{2}$, Paula Daniella de Abreu ${ }^{3}$, Ednaldo Cavalcante de Araújo ${ }^{4}$, Maria Lúcia Neto de Menezes ${ }^{5}$, Elaine Cristina Tôrres Oliveira ${ }^{6}$}

Resumo: Objetivo: avaliar a assistência pré-natal na perspectiva dos enfermeiros no âmbito da Estratégia Saúde da Família. Método: estudo descritivo, exploratório de abordagem quantitativa, realizado com 29 enfermeiros em 20 Estratégias Saúde da Família no ano de 2016. Analisou-se os dados coletados por meio de questionário com o auxílio do programa Statistical Package for Social Sciences for Personal Computer. Resultados: ressaltou-se que 55,2\% dos enfermeiros consideraram insuficientes os recursos humanos. Foi observado que $41,4 \%$ das unidades possuíam teste rápido para Ś́filis e Vírus da Imunodeficiência Humana; 69\% material para realização de exame ginecológico, sendo este realizado por $55,2 \%$ dos profissionais. Conclusão: a avaliação da assistência pré-natal, na perspectiva dos enfermeiros, apresentou contribuições para o reconhecimento dos limites e possibilidades para a adesão ao pré-natal conforme

\footnotetext{
${ }^{1}$ Enfermeira. Especialista em Saúde da Família pela Universidade de Pernambuco/UPE, Recife (PE), Brasil. E-mail: luanacarla6@hotmail.com ORCID: https://orcid.org/0000-0002-5569-7218

2 Enfermeira. Doutora em Saúde Pública pela Universidade Autônoma de Barcelona/UAB. Docente-pesquisadora da Universidade de Pernambuco (UPE), coordenadora do nó UPE, da Rede de pesquisas da África e América Latinas, Recife (PE), Brasil. E-mail: rejane.ferreira@upe.br - ORCID: https://orcid.org/0000-0002-7607-0502

${ }^{3}$ Enfermeira. Especialista em Saúde da Família pela Universidade de Pernambuco/UPE. Mestre em Enfermagem pelo Programa de PósGraduação em Enfermagem da Universidade Federal de Pernambuco/PPGENF-UFPE. Discente do Programa de Pós-Graduação de Enfermagem em Saúde Pública da Escola de Enfermagem de Ribeirão Preto da Universidade de São Paulo. Ribeirão Preto (SP), Brasil. E-mail: pauladdabreu@gmail.com - ORCID: https://orcid.org/0000-0001-8756-8173

${ }^{4}$ Enfermeiro. Doutor em Ciências pela Universidade Federal de São Paulo/UNIFESP. Docente do Departamento de Enfermagem da Universidade Federal de Pernambuco e do Programa de Pós-Graduação em Enfermagem (Mestrado Acadêmico e Doutorado)/UFPE/PPGENF, Recife (PE), Brasil. E-mail: ednaldo.araujo@ufpe.br - ORCID: https://orcid.org/0000-0002-1834-4544

${ }^{5}$ Enfermeira Obstetra. Doutora em saúde da criança e adolescente. Docente da Universidade de Pernambuco. FENSG/UPE, Recife (PE), Brasil. Email: maria.luciamenezes@yahoo.com.br - ORCID: https:// orcid.org/ 0000-0002-7161-8049

${ }^{6}$ Enfermeira. Doutoranda na Faculdade de Saúde Pública da Universidade de São Paulo/FSP-USP. Docente de Saúde Coletiva da Universidade Estadual de Ciências da Saúde de Alagoas/UNCISAL. Maceió (AL), Brasil. E-mail: laineoliv@hotmail.com - ORCID: https://orcid.org/0000-00021324-7163
} 
recomendado pelo Ministério da Saúde, com vistas à redução de riscos à saúde materno-fetal.

Descritores: Enfermagem; Cuidado pré-natal; Atenção primária à saúde; Saúde da família; Saúde pública

\begin{abstract}
Objective: to evaluate prenatal care from the perspective of nurses within the scope of the Family Health Strategy. Method: descriptive, exploratory study with a quantitative approach, conducted with 29 nurses in 20 Family Health Strategies in 2016. Data collected through a questionnaire was analyzed with the aid of the Statistical Package for Social Sciences for Personal Computer program. Results: it was emphasized that $55.2 \%$ of nurses considered human resources insufficient. It was observed that $41.4 \%$ of the units had a rapid test for Syphilis and Human Immunodeficiency Virus; $69 \%$ material for gynecological examination, which was performed by $55.2 \%$ of professionals. Conclusion: the assessment of prenatal care, from the nurses' perspective, made contributions to the recognition of the limits and possibilities for adherence to prenatal care as recommended by the Ministry of Health, with a view to reducing risks to maternal and fetal health.
\end{abstract}

Descriptors: Nursing; Prenatal care; Primary health care; Family Health; Public health

Resumen: Objetivo: Evaluar la asistencia prenatal en la perspectiva de los enfermeros en el ámbito de la Estrategia Salud de la Familia. Método: Estudio descriptivo, exploratorio de abordaje cuantitativo, realizado con 29 enfermeros en 20 Estrategias Salud de la Familia en el año de 2016. Se ha analizado los datos recogidos por medio de cuestionario con el auxilio del programa Statistical Package for Social Sciences for Personal Computer. Resultados: Se ha resaltado que $55,2 \%$ de los enfermeros consideraron insuficientes los recursos humanos. Ha sido observado que $41,4 \%$ de las unidades poseían test rápido para Sífilis y Virus de la Inmunodeficiencia Humana; 69\% material para realización de examen ginecológico, siendo esto realizado por 55,2\% de los profesionales. Conclusión: La evaluación de la asistencia prenatal, en la perspectiva de los enfermeros, presentó contribuciones para el reconocimiento de los límites y posibilidades para la adhesión al prenatal conforme recomendado por el Ministerio de la Salud, con el objetivo de la reducción de riesgos a la salud materno fetal.

Descriptores: Enfermería; Cuidado prenatal; Atención primaria a la salud; Salud de la familia; Salud pública

\title{
Introdução
}

A assistência ao pré-natal compreende um conjunto de ações direcionadas à saúde da mulher com o objetivo de reduzir a morbimortalidade materno-fetal. A dinâmica da assistência envolve a perspectiva biopsicossocial, sendo um evento que perpassa a dimensão biológica e implica no reconhecimento das condições fisiológicas, psicossociais, econômicas e acesso a informações de saúde, possibilitando a prevenção e tratamento precoce das alterações da gravidez, que são influenciadas pela história pessoal da gestante e por seu passado obstétrico, no qual o contexto da gravidez, a idade, o vínculo com o parceiro e o momento atual são essenciais para compreender o ciclo grávido-puerperal..$^{1-2}$ 
3 | Nascimento LCS, Silva MRF, Abreu PD, Araújo EC, Menezes MLN, Oliveira ECT

No Brasil o pré-natal adequado é mais frequente entre as mulheres de cor branca $(87,8 \%)$ e que realizaram o pré-natal na rede privada. As regiões do país que apresentam as menores frequências são: Norte $(69,5 \%)$ e Nordeste $(76,1 \%)$, respectivamente, enquanto a região Sudeste tem a maior frequência $(86,3 \%) .^{3}$

Mesmo diante da redução da mortalidade materna, no período entre 1990 e 2015, os números de óbitos no mundo ainda são alarmantes. De acordo com a Organização Pan-Americana de Saúde (OPAS), em 2018, aproximadamente 830 mulheres morreram diariamente em decorrência de eventos relacionados à gestação e ao parto, sendo 99,0\% destes óbitos em países de baixa e média renda. No Brasil, apesar da redução da mortalidade materna, a partir de 2016 houve um considerável aumento do número de casos. ${ }^{3} \mathrm{O}$ acompanhamento da gestante no âmbito da Saúde Pública demanda da identificação precoce de eventos indesejáveis, assistência multiprofissional e valorização do contínuo registro da assistência nos cartões de Pré-Natal da gestante. ${ }^{4}$

Com vistas a melhorar a saúde materna, a Organização das Nações Unidas (ONU), em 2015, lança uma agenda mundial que reúne objetivos para o desenvolvimento sustentável a serem atingidos até o ano de 2030. A perspectiva é que, a partir de ações integradas que abordam promoção da saúde e bem-estar, reduza-se, no âmbito da saúde materna, a taxa de mortalidade para menos de 70 mortes para cada 100 mil nascidos vivos, em todo o mundo. ${ }^{5}$

$\mathrm{Na}$ busca por atingir os objetivos pactuados mundialmente, como também garantir saúde à mulher no período gravídico-puerperal, no Brasil o pré-natal é um direito preconizado pelo Sistema Único de Saúde (SUS) e tem na Atenção Primária à Saúde (APS), preferencialmente com cobertura da Estratégia Saúde da Família (ESF), uma porta de entrada para o atendimento longitudinal à gestante de baixo risco, com consultas mensais até a $28^{\mathbf{a}}$ semana, quinzenais entre $28^{a}$ e $36^{a}$ semanas e semanais partir da $36^{a}$ até o parto.

Além disso, durante o pré-natal é garantido o direito do(a) parceiro(a) ser cuidado por 
Perspectiva dos enfermeiros sobre a assistência pré-natal no âmbito da Estratégia... | 4

meio da realização de consultas, exames laboratoriais e ter acesso às práticas de educação em saúde. Essa atenção à mulher contribui com o desfecho perinatal e materno favoráveis, em virtude da identificação dos fatores de risco que podem trazer complicações à gestação, além de permitir a detecção e o tratamento oportuno de complicações. ${ }^{6-7}$

Para que seja possível promover a saúde materna se faz necessário o cumprimento do número recomendado de consultas do pré-natal, para assegurar a prevenção, diagnóstico e tratamento das doenças intercorrentes da gestação e o estabelecimento de programa de imunização materna. Um pré-natal adequado se dá pela incorporação de condutas acolhedoras e construção de espaços para grupos de gestantes, para o compartilhamento de experiências, sentimentos, medos, expectativas e dúvidas, a fim de atender às principais queixas e demandas para promover um plano de cuidado individual e grupal de apoio..$^{6,8-9}$

Diante da necessidade de enfrentar problemas relacionados à assistência na gestação, no Brasil foi implantado o Programa de Humanização no Pré-natal e Nascimento (PHPN). Este programa objetiva assegurar a melhoria do acesso, da cobertura e da qualidade do acompanhamento, além da oferta da assistência ao parto e puerpério (às gestantes e ao recémnascido), para o alcance de uma atenção obstétrica integrada, qualificada e humanizada. ${ }^{10}$

De forma complementar à PHPN, o governo federal vem implementando desde 2011 o Programa Rede Cegonha, que tem por objetivo fomentar a implementação de um novo modelo de atenção à saúde da mulher e da criança, desde o parto até 24 meses de idade da criança. Procura-se organizar a Rede de Atenção à Saúde Materna e Infantil para garantir acesso, acolhimento, resolutividade e reduzir a mortalidade materna e infantil com destaque ao componente neonatal. ${ }^{10}$

Neste programa, o enfermeiro se apresenta em destaque quanto à assistência pré-natal de baixo risco, visto que atende a maioria das gestantes nas ESF. Diante disso, este estudo foi fundamento no pré-natal de baixo risco de acordo com o que é preconizado pelo Caderno de 
5 | Nascimento LCS, Silva MRF, Abreu PD, Araújo EC, Menezes MLN, Oliveira ECT

Atenção Básica (CAB) do Ministério da Saúde (MS), a fim de avaliar os atributos que impactam na redução de resultados obstétricos desfavoráveis. A qualidade do cuidado à gestante tem relação estreita com os níveis de saúde de mães e do bebê, sendo a sua investigação uma potente ferramenta norteadora para gestores e profissionais de saúde. ${ }^{6}$

Apesar da ampla cobertura, o pré-natal no Brasil ainda apresenta contexto de iniquidades e baixa oferta de atendimento adequado, especialmente entre mulheres negras que residem nas regiões mais pobres do país. ${ }^{3}$ Diante do exposto, é necessário avaliar a execução de atendimentos e ações que visem a integralidade do cuidado de forma equânime no âmbito do SUS e no contexto da região Nordeste do país. Desta forma, apresenta-se como questão de pesquisa: de que forma é ofertada a assistência pré-natal na perspectiva dos enfermeiros no âmbito da ESF? Este estudo teve como objetivo: avaliar a assistência pré-natal na perspectiva dos enfermeiros no âmbito da ESF.

\section{Método}

Trata-se de um estudo descritivo, exploratório de abordagem quantitativa, realizado no Distrito Sanitário II (DS II) da cidade do Recife, Pernambuco (PE), Brasil. A população foi composta por 42 enfermeiros lotados nas 20 ESF e a amostra constituída por 29 participantes (sete profissionais se recusaram a participar da pesquisa; quatro estavam afastados do serviço por licença médica e dois estavam de férias). Foram convidados a participar da pesquisa aqueles que atendessem ao seguinte critério de inclusão: exercer suas atividades nas ESFs pertencentes ao DSII. Foram excluídos aqueles que estavam de férias e os que estavam licença médica durante o período da pesquisa.

Para coleta de dados primários, foi utilizado um questionário elaborado pelos pesquisadores com perguntas abertas e fechadas, com total de 58 perguntas, elaboradas a partir do CAB: atenção ao pré-natal de baixo risco, editado pelo $\mathrm{MS},{ }^{6}$ com foco nas atribuições do profissional de enfermagem na atenção à gestante. 
Perspectiva dos enfermeiros sobre a assistência pré-natal no âmbito da Estratégia... 6

O questionário foi organizado considerando as seguintes variáveis: Perfil dos enfermeiros (idade, sexo, instituição de formação, cursos de pós-graduação e tempo de atuação na APS); Organização dos serviços, planejamento e programação (estrutura física das unidades, recursos humanos, os equipamentos e materiais disponíveis na ESF, o planejamento e programação das atividades); Acolhimento (forma de acolhimento da gestante e seu acompanhante); Atenção prénatal (diagnóstico da gravidez, classificação de risco gestacional, primeira consulta e subsequentes, avaliação do estado nutricional, exame ginecológico e coleta de materiais para colpocitologia oncótica, exame clínico das mamas) e Ações educativas (temas abordados e a forma que essas atividades são desenvolvidas).

A coleta de dados da pesquisa foi desenvolvida no período de outubro a novembro de 2016. O tempo de coleta se estendeu para 60 dias, porque algumas ESF precisaram ser visitadas mais de duas vezes, devido à dificuldade de localização do profissional de enfermagem, que se encontrava em visitas domiciliares ou em outras atividades.

A análise dos dados foi realizada por meio da construção de um banco de dados no programa Statistical Package for Social Sciences for Personal Computer (SPSS-PC), ${ }^{11}$ versão 22. A utilização desse programa teve como finalidade obter agilidade na interpretação e análises das informações coletadas. Após a codificação, os dados foram analisados estatisticamente a partir da qual se obteve as suas frequências relativas e absolutas. A apresentação das tabelas foi realizada com auxílio do programa Excel 2010.

A pesquisa foi norteada pelos aspectos éticos e as implicações legais de acordo com o Conselho Nacional de Saúde de nº. 466, de 12 de dezembro de 2012 o qual aprova as diretrizes e normas da pesquisa envolvendo seres humanos e aprovado pelo Comitê de Ética do Complexo Hospitalar Hospital Universitário Oswaldo Cruz, Pronto Socorro Cardiológico de Pernambuco, no dia 08/09/2016 com Parecer nº 1.716 .887 . 
7 | Nascimento LCS, Silva MRF, Abreu PD, Araújo EC, Menezes MLN, Oliveira ECT

\section{Resultados}

Os participantes do presente estudo apresentavam idade entre 26 e 65 anos, sendo a maioria pertencente a faixa etária de 26 a 45 anos (51,7\%). Houve predomínio de enfermeiros do sexo feminino (96,6\%), graduados em universidade pública (62,1\%), com titulação de especialistas/residentes (93,1\%).

Na Tabela 1 , observa-se que $75,9 \%$ dos respondentes mostraram-se insatisfeitos com a área física da ESF. Ao serem consultados quanto aos recursos humanos, mais da metade $(55,2 \%)$ respondeu que não são suficientes para o atendimento adequado. Quanto aos recursos materiais, 69,0\% dispõe de material para realização de exame ginecológico. Com relação à eficiência do sistema de referência e contra referência, $20,7 \%$ dos enfermeiros consideraram o sistema eficiente. Durante as consultas de pré-natal, 100,0\% dos participantes responderam que realizam classificação de risco. Quando perguntados se consultavam com frequência o CAB Prénatal de Baixo Risco, 34,5\% responderam que não. 
Perspectiva dos enfermeiros sobre a assistência pré-natal no âmbito da Estratégia... $\mid 8$

Tabela 1 - Distribuição da frequência de aspectos relacionados à adequação da organização dos serviços, do planejamento e programação das atividades de pré-natal. Distrito Sanitário II, Recife, Brasil, 2016.

\begin{tabular}{|c|c|c|}
\hline Variáveis & $\mathbf{n}$ & $\%$ \\
\hline \multicolumn{3}{|l|}{ Adequação da área física } \\
\hline $\operatorname{Sim}$ & 7 & 24,1 \\
\hline Não & 22 & 75,9 \\
\hline \multicolumn{3}{|l|}{ Recursos Humanos } \\
\hline $\operatorname{Sim}$ & 13 & 44,8 \\
\hline Não & 16 & 55,2 \\
\hline \multicolumn{3}{|l|}{ Recursos Materiais } \\
\hline Mesa Ginecológica & 29 & 100,0 \\
\hline Foco de Luz & 28 & 96,6 \\
\hline Esfigmomanômetro & 28 & 96,6 \\
\hline Balança para adulto & 27 & 93,1 \\
\hline Fita métrica flexível e inelástica & 22 & 75,9 \\
\hline Sonar Doppler & 22 & 75,9 \\
\hline Gestograma ou disco Obstétrico & 22 & 75,9 \\
\hline Material para exame ginecológico & 20 & 69,0 \\
\hline \multicolumn{3}{|l|}{$\begin{array}{l}\text { Eficiência do sistema de Referência e Contra } \\
\text { referência }\end{array}$} \\
\hline $\operatorname{Sim}$ & 6 & 20,7 \\
\hline Não & 23 & 79,3 \\
\hline \multicolumn{3}{|l|}{ Classificação de Risco Gestacional } \\
\hline $\operatorname{Sim}$ & 29 & 100,0 \\
\hline \multicolumn{3}{|l|}{ Uso do Caderno de Atenção Básica } \\
\hline $\operatorname{Sim}$ & 18 & 62,1 \\
\hline Não & 10 & 34,5 \\
\hline Não respondeu & 1 & 3,4 \\
\hline
\end{tabular}

Fonte: Dados primários

Com relação ao acolhimento imediato às gestantes, observou-se que 7,0\% dos participantes responderam que não o fazem. No que se refere ao acolhimento do acompanhante da mulher para participação no pré-natal, 96,6\% dos profissionais responderam que o fazem.

O Gráfico 1 mostra a distribuição dos itens relacionados à assistência pré-natal com relação à captação precoce e o diagnóstico da gravidez por meio da oferta de teste rápido de gravidez e solicitação do Beta Human Chorionic Gonadrotopin (BHCG). Observou-se que 82,2\% 
9 | Nascimento LCS, Silva MRF, Abreu PD, Araújo EC, Menezes MLN, Oliveira ECT

das gestantes foram captadas de forma precoce pelos profissionais e que em mais de $80,0 \%$ destas foram solicitados exames comprobatórios de gravidez.

Gráfico 1 - Distribuição das características da assistência pré-natal, com relação a captação precoce, e diagnóstico da gravidez nas ESF do DSII, Recife, 2016.

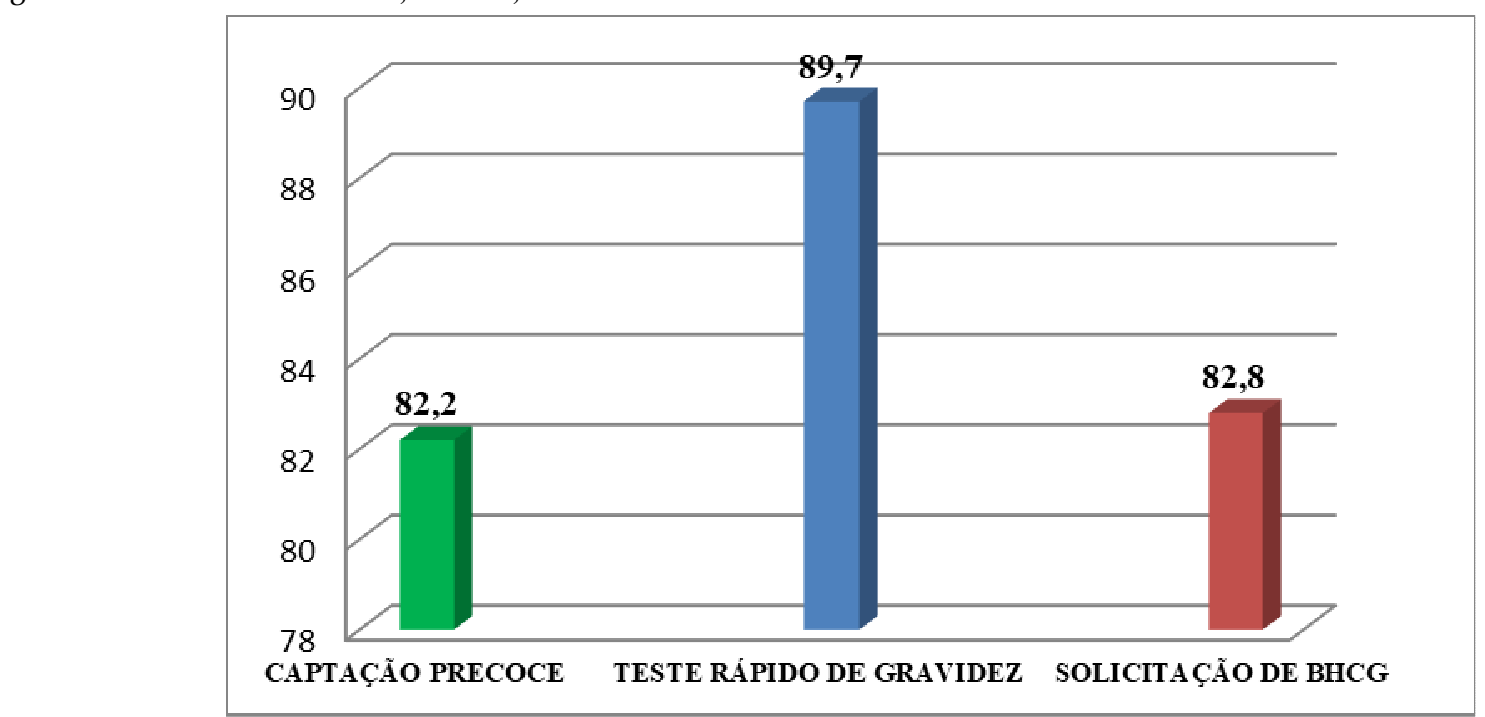

Fonte: dados primários

$\mathrm{Na}$ Tabela 2, encontram-se expostos os resultados referentes ao preenchimento da ficha clínica de pré-natal, registro da maternidade de referência no cartão da gestante, às consultas de pré-natal e à realização de exames preventivos. Observa-se que a maioria dos enfermeiros informou realizar o preenchimento da ficha clínica de pré-natal, solicitar exames complementares e fazer o registro da maternidade de referência $(93,1 \%, 93,1 \%$ e $79,3 \%$, respectivamente). 
Tabela 2 - Distribuição das características da assistência pré-natal prestada por enfermeiros nas ESF do DSII, Recife, 2016.

\begin{tabular}{lcc}
\hline & $\mathbf{n}$ & $\mathbf{\%}$ \\
& $(\mathrm{Sim})$ & $(\mathrm{Sim})$ \\
\hline Preenchimento da ficha Clínica PN & 27 & 93,1 \\
Solicitação de Exames Complementares & 27 & 93,1 \\
Registro da maternidade de Referência & 23 & 79,3 \\
Primeira Consulta & & \\
$\quad$ Anamnese & 28 & 96,6 \\
Suplementação vitamínica & 29 & 100,0 \\
Consultas Intercaladas com o Médico & 25 & 86,2 \\
Oferta teste rápido para sífilis/HIV & 12 & 41,4 \\
Consultas Subsequentes & & \\
Exame Físico direcionado & 29 & 100,0 \\
Controle de calendário vacinal & 29 & 100,0 \\
Avaliação de exames laboratoriais e outros & 29 & 100,0 \\
Avaliação do estado emocional & 29 & 100,0 \\
Busca ativa & 28 & 96,6 \\
Avaliação do estado nutricional & 25 & 86,2 \\
Exames Preventivos & & \\
$\quad$ Citologia oncótica & 16 & 55,2 \\
Exame clínico das mamas & 28 & 96,6 \\
\hline${ }^{*}$ HIV: Human Immunodeficiency Virus & & \\
Fonte: Dados primários & &
\end{tabular}

Com relação aos exames complementares solicitados na primeira consulta, conforme preconizado pelo CAB Pré-natal de Baixo Risco, os mais citados quanto a solicitação foram: hemograma $(86,2 \%)$, tipagem sanguínea e fator $\mathrm{Rh}(86,2 \%)$, glicemia de jejum $(86,2 \%)$, sorologias que incluem triagem para sífilis e ou Venereal Disease Research Laboratory (VDRL) (89,7\%), anti-HIV (Human Immunodeficiency Virus) (89,7\%), toxoplasmose (86,2\%), sorologia para hepatite B (HbsAg) (82,8\%) e Sumário de urina (82,8\%). De forma menos frequente foram citados: urocultura $(75,9 \%)$, eletroforese da hemoglobina $(58,6 \%)$, ultrassonografia obstétrica $(51,7 \%)$ e exame parasitológico de fezes $(27,6 \%)$. O questionamento sobre os exames complementares não foi respondido por $10,3 \%$ dos participantes. 
Em relação ao exame físico direcionado e específico, clínico e obstétrico, verificou-se que $100 \%$ dos enfermeiros referem sua realização. Entre os procedimentos feitos com maior frequência estão o controle da pressão arterial, a palpação obstétrica e medida de altura uterina, ausculta de batimentos cardiofetais e verificação de edema. Quando questionados sobre quais atividades educativas, $89,7 \%$, dos enfermeiros referiram que realizam atividades individuais e $13,8 \%$ dos entrevistados não responderam sobre as atividades em grupos e sala de espera conforme descrito na Tabela 3.

Tabela 3 - Distribuição das atividades educativas e temas abordados nos grupos nas Unidades de Saúde da Família do Distrito Sanitário II, Recife-PE, Brasil 2016.

\begin{tabular}{lcc}
\hline Variável & $\mathbf{n}$ & $\%$ \\
\hline Atividades educativas & & \\
Individuais & 26 & 89,7 \\
$\quad$ Sim & 3 & 10,3 \\
Não & & \\
Em grupo e sala de espera & 15 & 51,7 \\
Sim & 10 & 34,5 \\
Não & 4 & 13,8 \\
Não respondeu & & \\
Temáticas das atividades educativas & 29 & 100,0 \\
Aleitamento materno & 28 & 96,6 \\
Hábitos saudáveis & 27 & 93,1 \\
Planejamento familiar & 27 & 93,1 \\
Cuidados com o Recém-nascido & 26 & 89,7 \\
Importância do pré-natal & 26 & 89,7 \\
Sinais de Trabalho de parto & 25 & 86,2 \\
Riscos associados à gestação e parto & 25 & 86,2 \\
Riscos de automedicação & 25 & 86,2 \\
Riscos de álcool e drogas & 24 & 82,8 \\
Atividade sexual e prevenção de IST/Aids* & 22 & 75,9 \\
Direitos da gestante e do pai & 16 & 55,2 \\
Violência doméstica e sexual & & \\
\hline
\end{tabular}

*IST: Infecções Sexualmente Transmissíveis / Aids: Síndrome da Imunodeficiência Adquirida Fonte: dados primários

Entre as várias temáticas educativas que devem ser abordadas no pré-natal destacaramse: orientações quanto ao aleitamento materno (100\%), seguidas das orientações referentes aos hábitos saudáveis, planejamento familiar, os cuidados com recém-nascido, a importância pré- 
natal, entre outros. Já os temas como direitos da gestante e do pai e violência doméstica e sexual foram os menos abordados (Tabela 3).

\section{Discussão}

No presente estudo foi possível analisar a qualidade do processo da assistência pré-natal no município do Recife sob a ótica de enfermeiros das ESF localizadas no DSII. Para tal, foi utilizado como referencial as preconizações dispostas no CAB Pré-natal de Baixo Risco que versa sobre a organização do processo de trabalho, do serviço de saúde e aspectos do planejamento, estabelecidos pelo MS para o acompanhamento de gestantes de baixo risco gestacional e de possíveis intercorrências. Além disso, foram avaliados os aspectos referentes à promoção da saúde, gestações especiais e os aspectos legais relacionados à gestação, ao parto/nascimento e ao puerpério. ${ }^{10}$

Nessa pesquisa a maioria dos participantes foram do sexo feminino. É evidente o predomínio feminino na enfermagem e o contexto da participação da mulher nas atividades do cuidado em saúde. Este aspecto pode estar relacionado com a inserção desta no mercado de trabalho e as possibilidades de atividades fora do âmbito doméstico, dada a natureza assistencial e papel de cuidadora destinado historicamente pela sociedade. ${ }^{12}$

No cenário estudado foi identificada a baixa adesão ao Programa Nacional de Melhoria do Acesso e da Qualidade da Atenção Básica (PMAQ). A pequena aderência aos focos estratégicos do pré-natal, às iniciativas e programas estratégicos do MS, como a Rede Cegonha e o Pacto pela Vida, pode contribuir com riscos de morbimortalidade e desfechos desfavoráveis materno e infantil que poderiam ser evitados. ${ }^{13}$

Em relação à organização dos serviços, planejamento e programação, obteve-se destaque a falta de infraestrutura adequada e recursos humanos insuficientes. Essas dificuldades aludidas por esses enfermeiros podem reduzir a qualidade do atendimento à população, visto que elas 
dificultam a assistência, o acompanhamento de forma integral e a resolução dos problemas. Além disso, a inadequação de alguns espaços físicos, sua indisponibilidade, devido ao baixo quantitativo de salas para atendimento e espaços limitados para atividades grupais, são fatores que dificultam a qualidade da assistência. ${ }^{14}$

Estudo realizado à nível nacional evidenciou inadequação da infraestrutura da rede de atenção básica que oferta o pré-natal em todas as regiões geopolíticas do Brasil. Além disso, referiu a precarização de adequação de ações clínicas para a qualidade do cuidado e insuficiente capacidade de gestão das equipes para a garantia do acesso. Também foi referido uma relação direta entre adequação da infraestrutura e contextos sociais com Índice de Desenvolvimento Humano Municipal e renda mais elevados. ${ }^{15} \mathrm{O}$ acompanhamento da gestante e binômio mãe/filho ocorre de forma longitudinal, sendo o ambiente adequado à realização do exame físico detalhado, durante as consultas do enfermeiro, estratégia fundamental para reduzir a morbimortalidade materna e neonatal. ${ }^{8}$

Com relação aos equipamentos e materiais, as unidades de saúde devem dispor minimamente de: mesa e cadeiras para a realização das consultas; mesa ginecológica; escada de dois degraus; foco de luz; balança antropométrica para adultos; esfignomanômetro; estetoscópio clínico; estetoscópio de Pinard; fita métrica; espéculos; pinças de Cheron; instrumentos para coleta de material para exame colpocitológico; gestograma ou disco obstétrico; e sonar Doppler (se possível). ${ }^{6}$

Os dados referentes aos equipamentos materiais demostraram que a maioria das ESF dispõem dos materiais conforme preconizado pelo MS, e em 24,7\% havia a ausência de sonar Doppler, de estetoscópio de Pinard $(37,9 \%)$ e de materiais de coleta para colpocitologia oncótica (CCO) (31\%). Corroborando com um estudo do Ceará, em que foram avaliadas as concepções de gestantes e enfermeiros acerca da assistência pré-natal na APS, foi observada a carência de materiais nas ESF, para verificar batimentos cardíacos fetais. ${ }^{7}$ A maioria das ESF possuem duas equipes e os atendimentos ao pré-natal são agendados, devido a indisponibilidade do aparelho 
Perspectiva dos enfermeiros sobre a assistência pré-natal no âmbito da Estratégia... | 14

para cada equipe, as equipes tem que dividir, o profissional tem quer sair da sala e pegar o sonar com o outro profissional que também está em atendimento.

Considerando as questões relativas à classificação de risco gestacional, o manejo pode ser considerado elevado, uma vez que todos os profissionais o realizavam, corroborando com a literatura. ${ }^{15} \mathrm{O}$ MS orienta que a classificação do risco gestacional deve ser realizada na primeira consulta e nas subsequentes, além de ser indispensável garantir o acesso ao serviço de referência para as gestantes classificadas como de risco. ${ }^{10}$

Com relação ao mecanismo de coordenação da assistência no sistema de referência e contra-referência, merece destaque a baixa eficiência referida pelos enfermeiros, com relação ao atendimento às gestantes. Os resultados encontrados corroboram com outros estudos. ${ }^{15-16} \mathrm{~A}$ baixa eficiência do processo de referência e contra-referência é um indicativo de falhas na dinâmica de encaminhamentos e retorno das informações pelos especialistas que participam da rede de atendimento, assim, são necessárias melhorias na gestão deste processo de modo a garantir eficácia do acompanhamento longitudinal das gestantes durante o pré-natal. ${ }^{17}$

A implantação do prontuário eletrônico pode viabilizar a assistência pré-natal, sobretudo para o sistema de referência e contra-referência, pois permite o fluxo de informações entre os serviços de saúde dos diversos níveis de atenção. ${ }^{16}$ Nesse sentido, deve-se garantir o vínculo entre a ESF e os demais centros de referência da saúde para a assistência integral da gestante durante o pré-natal, parto e puerpério, no âmbito da atenção primária e em maternidades, conforme definição do gestor local.

Além disso, torna-se imprescindível que todas as gestantes sejam orientadas/encaminhadas aos atendimentos especializados quando apresentarem intercorrências clínicas/obstétricas ou quando estiver em trabalho de parto. Estes núcleos de atenção à saúde devem prestar a contra-referência para a unidade de origem para continuidade da assistência, de forma integral e longitudinal pela ESF de seu território. 
15 | Nascimento LCS, Silva MRF, Abreu PD, Araújo EC, Menezes MLN, Oliveira ECT

Sobre a utilização regular durante as consultas do CAB Atenção ao Pré-natal de Baixo Risco, foi observado que para a maioria dos enfermeiros este documento se constitui um instrumento importante para a condução do cuidado. Ressalta-se que a maioria dos profissionais possuíam especialização no campo da saúde pública e atuam há mais de 11 anos nas ESF. O embasamento teórico-prático a partir da assistência padronizada, com consulta ao $\mathrm{CAB}$, são fundamentais para a assistência integral. ${ }^{10}$

O MS reconhece a estrutura organizacional como ponto fundamental para garantir uma prática segura durante a assistência pré-natal. Nessa perspectiva, é recomendado um ambiente facilitador para as ações integradas de saúde que considerem aspectos relacionados à planta física, aos recursos humanos e materiais, ao apoio laboratorial, ao acesso ao medicamento, aos instrumentos de registro e ao sistema de referência e contra-referência. ${ }^{15}$

A ambiência na saúde refere-se ao tratamento dado ao espaço físico, entendido como espaço social, profissional e de relações interpessoais que deve proporcionar atenção acolhedora, resolutiva e humana. Os dados apresentados mostram a insatisfação com relação a ambiência das ESFs, uma vez que a maioria não se localiza em prédios adequados, com comprometimento da infraestrutura, poucos consultórios para atendimento e espaços limitados para ações de promoção da saúde.

Nos resultados observou-se que $93,1 \%$ dos profissionais faziam o acolhimento imediato de todas as gestantes e puérperas. A acolhida rápida é garantia de atendimento de todos os recém-nascidos e à totalidade da gestante e puérperas que procurem a ESF, preconizado para uma assistência pré-natal efetiva. ${ }^{6}$

O acolhimento, conforme preconizado na política de humanização, implica na recepção da mulher, desde sua chegada à ESF. ${ }^{16}$ Assim, a construção do vínculo é importante para um pré-natal satisfatório. Acolher é, antes de tudo, uma ação ética a ser exercida por todos os integrantes da equipe multiprofissional para escuta qualificada, a fim de atender às 
Perspectiva dos enfermeiros sobre a assistência pré-natal no âmbito da Estratégia... | 16

necessidades da gestante. Está ação também implica no fluxo dos processos de trabalho, das equipes, dos serviços e das redes, direcionada à concepção da clínica ampliada.

Considerando a captação precoce, o diagnóstico da gravidez por meio da oferta de teste rápido e solicitação do BHCG obteve resultados satisfatórios com relação a adequação da assistência pré-natal. Essa assistência inclui o diagnóstico da gravidez, seja pela oferta de teste rápido, ou pela solicitação do BHCG diante dos sinais de presunção e de probabilidade apresentados pela paciente, estes testes foram implantados pelo MS, por intermédio da rede cegonha, para ampliar a captação precoce das gestantes, pois acelera o processo necessário para a confirmação da gravidez e o início do pré-natal. ${ }^{6}$

Nessa pesquisa a frequência de registros da maternidade de referência referida no cartão das gestantes foi superior a 79\%. Em outra pesquisa, foi evidenciado registros semelhantes, $87,3 \%$ das puérperas relataram ter recebido informações sobre a maternidade de referência. A omissão dessa orientação pode levar a uma jornada das gestantes pelos estabelecimentos de saúde no momento do parto ou em caso de emergências. ${ }^{15}$

O rastreamento das Infecções Sexualmente Transmissíveis (IST) no pré-natal assumem alta relevância. A detecção do HIV e da sífilis durante o pré-natal é imprescindível para o controle da transmissão vertical..$^{15}$

É recomendado que as consultas de pré-natal baixo risco sejam intercaladas entre o médico e o enfermeiro. ${ }^{3}$ Nos resultados evidenciou-se que $13,8 \%$ dos profissionais referiram que não haviam consultas intercaladas com médico, no entanto, o trabalho em equipe no acompanhamento pré-natal na atenção básica contribui com o compartilhamento de saberes e integralidade da assistência. Assim, as consultas intercaladas poderiam favorecer a tomada de decisões para o melhor direcionamento das consultas, solicitação de exames complementares, discussão de caso, além de ações educativas com temáticas que visem a redução da morbimortalidade materna e infantil. 
No que se refere ao exame de Colpocitologia, os resultados encontrados apresentaram que $44,8 \%$ dos profissionais não o realiza durante a gestação. De acordo com o MS este exame deve ser realizado preferencialmente $7^{\circ}$ mês de gestação conforme indicação individualizada. ${ }^{6}$

A prevenção ou diagnóstico do câncer de colo uterino durante a gravidez é recomendada pelo MS, sobretudo para as mulheres com atraso no acompanhamento preventivo. Porém, muitas mulheres ainda não se submetem a realização do exame na gestação, por vergonha ou medo, constrangimento, dificuldade de comunicação, desconforto entre outros fatores e por isso, podem perder a oportunidade de prevenção. ${ }^{6}$

A realização do exame das mamas é importante durante a gravidez, pois ele permite a observação de alterações e transformações durante o período gravídico. Modificações tais como aumento da pigmentação da aréola e mamilo, presença de veias superficiais visíveis, presença de glândulas sebáceas na aréola, colostro e anomalias no que possam dificultar a lactação, entre outros, devendo assim ser praticado desde a primeira consulta. ${ }^{6}$ Outro estudo realizado na região Nordeste identificou que $50 \%$ dos enfermeiros realizaram o exame das mamas das gestantes. ${ }^{8}$

Foi evidenciado que $90 \%$ das gestantes estavam satisfeitas com o atendimento do enfermeiro. No entanto, é primordial a realização de outras pesquisas que considerem uma análise empírica acerca da realização do pré-natal, mediante a educação permanente com vistas à qualificação da assistência por meio da ação-reflexão-ação das suas práticas. ${ }^{18}$

O enfermeiro também deve estar apto a realização de visitas domiciliares que visem à ampliação da assistência ao PN, quando necessárias, para evitar possíveis complicações. Para que haja uma melhoria de qualidade da assistência pré-natal, é importante a oferta da educação permanente por gestores, com ênfase nas competências essenciais da assistência ao pré-natal. ${ }^{15-}$ ${ }^{17}$ A implementação de estratégias de educação permanente em saúde deve priorizar o caráter crítico-reflexivo da assistência ancorada aos preceitos da APS, pactuada com a Rede Cegonha e aos saberes e práticas da equipe multiprofissional com vistas a qualidade do cuidado integral 
durante o pré-natal. ${ }^{19}$

\section{Conclusão}

A avaliação da assistência pré-natal, na perspectiva dos enfermeiros no âmbito ESF, apresentou contribuições para o reconhecimento dos limites e possibilidades para a adesão ao pré-natal. Essa adesão, conforme recomendado pelo MS, visa à redução de riscos à saúde materno-fetal, considerando o contexto de vulnerabilidade social de um distrito sanitário da região nordeste do Brasil.

Foram evidenciadas a qualidade insatisfatória da assistência pré-natal, decorrente da infraestrutura inadequada, recursos humanos e materiais insuficientes, sistemas de referência e contra-referência ineficazes, além da demora e a não realização dos exames preconizados. Tais achados remetem à determinação social do processo saúde-doença e à necessidade de corresponsabilização do Estado, profissionais da saúde e usuários da saúde no enfrentamento da morbimortalidade materna e perinatal.

A qualificação permanente do enfermeiro para a assistência pré-natal deve possibilitar uma experiência satisfatória e humanizada, que considere os significados da maternidade e oportunize a promoção da saúde e prevenção de doenças e agravos.

Uma possível limitação foi a realização da coleta de dados em apenas um DS, com ênfase nos enfermeiros e nos aspectos relacionados ao pré-natal desta área. Em contrapartida, os resultados viabilizaram a avaliação da qualidade da assistência dos participantes, que possibilitou comparações com outros estudos. Recomenda-se a realização de novas pesquisas relacionadas à importância das práticas de educação permanente para os enfermeiros para a assistência qualificada no pré-natal.

\section{Referências}


1. Costa FJLS, Camara JT, Costa KR, Serejo ECS, Pedrosa AO, Lima AKA. Avaliação da assistência prénatal na perspectiva da integralidade. J. res.: fundam. care. [Internet] 2016 abr./jun. [acesso em 2020 mar 09] 8(2):4563-4586. Disponível em: http://seer.unirio.br/index.php/cuidadofundamental/article/view/5034 10.9789/21755361.2016.v8i2.4563-4586

2. Reis PAGD, Pereira CCA, Leite IC, Theme-Filha MM. Fatores associados à adequação do cuidado pré-natal e à assistência ao parto em São Tomé e Príncipe, 2008-2009. Cad Saúde Pública. [Internet] 2015 Mar [acesso em 2019 Jul 20];31(9):1929-40. Disponível em: http://www.scielo.br/pdf/csp/v31n9/0102-311X-csp-31-9-1929.pdf doi: 10.1590/0102-311X00115914.

3. Organização Pan-Americana da Saúde (OPAS). Brasília: Organização Mundial da Saúde. Folha informativa - Mortalidade materna. [internet] 2018 [acesso em 2019 Nov 09]. Disponível em: https://www.paho.org/bra/index.php?option=com_content\&view=article\&id=5741:folha-informativamortalidade-materna\&Itemid $=820$

4. Santos TMMG, Abreu APSB, Campos TG. Avaliação dos registros no cartão de pré-natal da gestante. Rev Enferm UFPE On Line [Internet]. 2017 [acesso em 2019 Nov 09];11(Supl 7):2939-45. Disponível em: https:/periodicos.ufpe.br/revistas/revistaenfermagem/article/viewFile/8458/19206 doi: 10.5205/reuol.11007-98133-3-SM.1107sup201715

5. Nações Unidas Brasil. Objetivos de Desenvolvimento Sustentável [Internet]. Brasília: Nações Unidas Brasil; 2015 [acesso em 2019 Nov 07]. Disponível em: https://nacoesunidas.org/pos2015

6. Ministério da Saúde (BR). Atenção ao pré-natal de baixo risco. Brasília: Ministério da Saúde. Cadernos de Atenção Básica, №32, de 2012. Série A. Normas e Manuais Técnicos. [internet] 2012 [acesso em 2018 Dez 11]. Disponível em: http://bvsms.saude.gov.br/bvs/publicacoes/cadernos_atencao_basica_32_prenatal.pd

7. Warmling CM, Fajardo AP, Meyer DE, Bedos C. Práticas sociais de medicalização \& humanização no cuidado de mulheres na gestação. Cad Saúde Pública. [Internet] 2018 Mar [acesso em 2019 Jul 20];34(4):e00009917. Disponível em: http://www.scielo.br/pdf/csp/v34n4/1678-4464-csp-34-04e00009917.pdf doi: 10.1590/0102-311X00009917

8. Lima LFC, Davim RMB, Silva RAR, et al. Importância do exame físico da gestante na consulta do enfermeiro. Rev Enferm UFPE On Line [Internet]. 2014 [acesso em 2019 Jul 20];8(6):1502-9. Disponível em: https://periodicos.ufpe.br/revistas/revistaenfermagem/article/viewFile/9838/10039 doi: 10.5205/reuol.5876-50610-1-SM.0806201407

9. Queiroza MVO, Menezesa GMD, Silva TJP, Brasil EGM, Silva RM. Grupo de gestantes adolescentes: contribuições para o cuidado no pré-natal. Rev Gaúcha Enferm. [Internet] 2016 Mar [acesso em 2019 Jul 20];37(esp):e2016-0029. Disponível em: http://www.scielo.br/scielo.php?script=sci_arttext\&pid=S1983-14472016000500418 doi: 10.1590/19831447.2016.esp.2016-0029 
Perspectiva dos enfermeiros sobre a assistência pré-natal no âmbito da Estratégia... $\mid 20$

10. Ministério da Saúde (BR). Humanização do parto e do nascimento. Brasília: Ministério da Saúde. Cadernos HumanizaSUS. [internet] 2014 [acesso em 2018 Dez 11]. Disponível em: http://www.redehumanizasus.net/sites/default/files/caderno_humanizasus_v4_humanizacao_parto.pdf

11. SPSS. Statistical Package for Social Sciences for Personal Computer. SPSS for Windows: Base System User̉ s Guide, Release 6,0. United States of America: SPSS Inc., 1993.

12. Costa CGS, Vieira DVF, Martins LHFA, Castro Júnior AR. Construção da imagem profissional no Ceará: a enfermeira diante das modificações no cenário histórico profissional. Cad. Saúde Colet. [Internet] 2019 [Internet] 2017 Mar [acesso em 2020 Abr 07];27(2):166-171. Disponível em: http://www.scielo.br/scielo.php?pid=S1414-462X2019000200166\&script=sci_arttext doi: 10.1590/1414462X201900020116

13. Tomasi E, Fernandes PAA, Fischer T, Siqueira FCV, Silveira DS, Thumé E, et al. Qualidade da atenção pré-natal na rede básica de saúde do Brasil: indicadores e desigualdades sociais. Cad. Saúde Pública. [Internet] 2017 Mar [acesso em 2019 Jul 20];33(3):e00195815. Disponível em: http://www.scielo.br/pdf/csp/v33n3/1678-4464-csp-33-03-e00195815.pdf $\quad$ doi: 10.1590/0102$311 \times 00195815$

14. Cunningham SD, Grilo S, Lewis JB, Novick G, Rising SS, Tobin JN, et al. Group Prenatal Care Attendance: Determinants and Relationship with Care Satisfaction. Matern Child Health J. [Internet] 2017 Mar $\quad$ acesso em 2019 Jul 20];21(4):770-76. Disponível em: https://www.ncbi.nlm.nih.gov/pmc/articles/PMC5290265/ doi: 10.1007/s10995-016-2161-3

15. Guimarães WSG, Parente RCP, Guimarães TLF, Garnelo L. Acesso e qualidade da atenção pré-natal na Estratégia Saúde da Família: infraestrutura, cuidado e gestão. Cad Saúde Pública. [Internet] 2018 [acesso em $2018 \mathrm{dez} 15]$; 34(5):e00110417 Disponível em: http://www.scielo.br/pdf/csp/v34n5/1678-4464csp-34-05-e00110417.pdf doi: 10.1590/0102-311X00110417

16. Dantas DS, Mendes RB, Santos JMJ, Valença TS, Mahl C, Barreiro MSC. Qualidade da Assistência Pré-Natal no Sistema Único de Saúde. Rev enferm UFPE on line [Internet] 2018 Maio [acesso em 2020 Fev 25];12(5):1365-71. Disponível em: https://periodicos.ufpe.br/revistas/revistaenfermagem/article/view/230531 doi: 10.5205/1981-8963v12i5a230531p1365-1371-2018

17. Mendes RB, Santos JMJ, Prado DS, et al. Características maternas e da assistência pré-natal associadas à peregrinação no anteparto. Rev Saude Publica. [Internet] 2019 [acesso em 2020 Jan 11]; 53:70. Disponível em: http://www.scielo.br/scielo.php?script=sci_arttext\&lng=pt\&tlng=pt\&pid=S003489102019000100258

18. Gonçalves MD, Kowalski ISG, Sá AC. Atenção ao pré-natal de baixo risco: atitudes dos enfermeiros da estratégia saúde da família. Rev enferm UERJ. [Internet] 2016 Mar [acesso em 2019 Jul 20];24(6):e18736. Disponível em: http://www.facenf.uerj.br/v24n6/v24n6a11.pdf doi: 10.12957/reuerj.2016.18736 
19. Leal NJ, Barreiro MSC, Mendes RB, Freitas CKAC. Prenatal care: nurses' testimonial. Rev Fund Care Online. [Internet] 2018 Mar [acesso em 2019 Jul 20];10(1):113-22. Disponível em: http://www.seer.unirio.br/index.php/cuidadofundamental/article/view/5991 doi: $\quad$ 10.9789/2175$\underline{5361.2018 . v 10 \mathrm{i} 1.113-122}$

\section{Autor correspondente}

Paula Daniella de Abreu

E-mail: pauladdabreu@gmail.com

Endreço: Av. Prof. Moraes Rego, 1235 - Cidade Universitária, Recife - PE

CEP: 50670-901

\section{Contribuições de Autoria}

\section{1 - Luana Carla dos Santos Nascimento}

Construção do projeto, condução da coleta e análise dos dados, bem como revisão crítica da versão final do relatório de pesquisa.

\section{2 - Maria Rejane Ferreira da Silva}

Suporte e orientação na construção do projeto, condução da coleta e análise dos dados, bem como revisão crítica da versão final do relatório de pesquisa.

\section{3 - Paula Daniella de Abreu}

Suporte e orientação na construção do projeto, condução da coleta e análise dos dados, bem como revisão crítica da versão final do relatório de pesquisa.

\section{4 - Ednaldo Cavalcante de Araújo}

Suporte e orientação na construção do projeto, condução da coleta e análise dos dados, bem como revisão crítica da versão final do relatório de pesquisa.

\section{5 - Maria Lúcia Neto de Menezes}

Construção do projeto, condução da coleta e análise dos dados, bem como revisão crítica da versão final do relatório de pesquisa.

\section{6 - Elaine Cristina Tôrres Oliveira}

Construção do projeto, condução da coleta e análise dos dados, bem como revisão crítica da versão final do relatório de pesquisa.

\section{Como citar este artigo}

Nascimento LCS, Silva MRF, Abreu PD, Araújo EC, Menezes MLN, Oliveira ECT. Perspectiva dos enfermeiros sobre a assistência pré-natal no âmbito da Estratégia Saúde da Família. Rev. Enferm. UFSM. 2020 [Acesso em: Anos Mês Dia]; vol.10 e34: 1-21. DOI:https://doi.org/10.5902/2179769238444 